\title{
EP-55
}

\section{Shorter operation time and improved surgical outcomes in laparoscopic donor right hepatectomy compared to open donor right hepatectomy: A single surgeon study}

Eui Soo HAN, Kwang-Woong LEE*, Kyung-Suk SUH, Nam-Joon YI, YoungRok CHOI, Suk Kyun HONG, Jeong-Moo LEE, Kwang Pyo HONG, Sanggyun SUH, Su young HONG

Department of Surgery, Seoul National University College of Medicine, Seoul, Korea

Introduction: Pure laparoscopic donor right hepatectomy (PLDRH) is a complex procedure, and the safety and feasibility of this operation remain unclear. This study aimed to evaluate the clinical outcomes and learning curve of this operation performed by a single surgeon.

Methods: We retrospectively reviewed the initial 100 donors who underwent PLDRH or open donor right hepatectomy (ODRH) by a single surgeon from December 2012 to May 2019. Endpoints analysed included intraoperative results, postoperative complications, and learning curve, which was evaluated using the cumulative sum method based on the operation time. We divided the PLDRH group into initial and recent groups based on the time point of overcoming the learning curve.

Results: The operative time was significantly shorter in the recent PLDRH group ( $\mathrm{n}=57 ; 181.0 \pm 35.7$ minutes) than in the ODRH ( $\mathrm{n}=$ 50; $203.0 \pm 37.3$ minutes) and initial PLDRH ( $n=43 ; 282.2 \pm 59.2$ minutes) groups $(p<0.001)$. Moreover, the length of hospital stay in the recent PLDRH group was significantly reduced compared to that in the ODRH group $(7.7 \pm 1.2$ vs. $5.8 \pm 1.4 ; p<0.001)$. The complication rate was reduced from $10 \%$ in the ODRH group and $8 \%$ in the initial PLDRH group to $2 \%$ in the recent PLDRH group.

Conclusions: If overcoming the learning curve is achieved by technical improvements, the operation time of PLDRH can be shorter and surgical outcomes are feasible compared to those of open donor hepatectomy. 